\title{
Prognostic impact of NOTCH1 and MYD88 mutations in chronic lymphocytic leukemia patients
}

\author{
Ewelina Zakrzewska ${ }^{1} \odot$, Agnieszka Karczmarczyk ${ }^{3} \odot$, Joanna Purkot ${ }^{3} \odot$, \\ Paulina Własiuk $^{2,3}{ }^{\oplus}$, Krzysztof Giannopoulos ${ }^{2,3}{ }^{\oplus}$, Elżbieta Starosławska ${ }^{1}$ \\ ${ }^{1}$ Medical Diagnostic Laboratory, St John's Cancer Center, Lublin \\ ${ }^{2}$ Department of Hematology, St John's Cancer Center, Lublin \\ ${ }^{3}$ Department of Experimental Hematooncology, Medical University of Lublin
}

This work was supported by Medical University of Lublin Scientific Grant MNmb537

\begin{abstract}
Summary
Background. Chronic lymphocytic leukemia (CLL) is one of the most common types of leukemia in adults with highly heterogeneous clinical course of the disease. Currently available prognostic factors are not fully efficient in predicting the course of CLL. New molecular mutations such as NOTCH1 and MYD88 could partly explain the CLL heterogeneity and help in identifying clinically relevant groups of patients.

Material and methods. NOTCH1 c.7544_7545delCT $(n=200)$ in PEST domain (exon 34) and MYD88 L265P $(n=60)$ mutations was investigated by amplification refractory mutation system (ARMS). Expression of MYD88 in CLL was assessed in peripheral blood (PB) $(n=60)$ and bone marrow (BM) $(n=92)$ of CLL patients and 25 healthy volunteers (HVs) using $q R T-P C R$.

Results. NOTCH1 mutation occurred in 18/200 (9.0\%) CLL patients. Patients harboring NOTCH1 mutations prevalently belonged to aggressive cases, i.e. cases with an unmutated IGVH gene status, expression of CD38 and ZAP-70. MYD88 mutation occurred in 2/60 (3.3\%) CLL patients. MYD88 mutations were strikingly enriched among patients expressing mutated IGVH genes. Our study demonstrated significantly higher PB MYD88 expression than in HVs and relevantly higher PBMYD88 expression in comparison with BM (respectively $p<0.0001$ and $p=0.0015)$. There was no correlation between MYD88 expression in PB and $B M$ and expression of ZAP-70, CD38 and IGVH mutational status.

Conclusions. NOTCH1 mutations are more frequently detected in cases with unfavorable biological markers and seem to be independent predictive markers for worse outcome in CLL patients. Further collaborative studies in CLL are obligate to study the prognostic and predictive relevance of MYD88 mutations and expression.
\end{abstract}

Key words: chronic lymphocytic leukemia, NOTCH1, MYD88, prognostic factors, ARMS-PCR

J. Transf. Med. 2019; 12: 101-108

Correspondence address: Dr n. med. Ewelina Zakrzewska, Medical Diagnostic Laboratory, St John's Cancer Center, Lublin, Jaczewskiego 7, 20-090 Lublin, phone: 5097085 05, fax: 8174789 11; e-mail: evelin84@wp.pl 


\section{Introduction}

Chronic lymphocytic leukemia (CLL) is one of the most common types of leukemia in adults and is characterized by the accumulation of malignant B CD5+ lymphocytes in the peripheral blood (PB) and lymphoid organs [1]. The clinical course of the disease is highly heterogeneous, from patients with stable disease to rapid progression leading to death within a year of diagnosis or to transformation to an aggressive lymphoma, known as Richter's syndrome (RS) [2-5]. Clinical heterogeneity of CLL explains the need for identification of prognostic and predictive factors.

Currently available prognostic factors are not fully efficient in predicting the course of CLL, especially when the disease is diagnosed at an early stage. Therefore, we are looking for new prognostic and predictive factors, which allow the classification of patients into the groups of disease progression at the time of diagnosis. The currently used prognostic factors in the early clinical stages are based on biological parameters of B-cell leukemias such as the mutational status of $I G V H$ (immunoglobulin heavy chain variable region) gene, gene aberrations, as well as expression of CD38 and ZAP-70 on B lymphocytes surface [6]. Moreover, Döhner et al. [7] used interphase fluorescence in situ hybridization (FISH) cytogenetic analysis to evaluate cytogenetic lesions in CLL, detecting chromosomal abnormalities in over $80 \%$ of patients. By correlating FISH changes with the course of the disease, a hierarchical model based on five risk categories was developed.

Recently, the use of next-generation sequencing (NGS) technologies has revealed previously unknown genomic alterations in this disease that are present at $5 \%$ to $15 \%$ (e.g. neurogenic locus notch homolog protein 1 [NOTCH1], splicing factor 3B subunit $1[S F 3 B 1]$, ataxia-telangiectasia mutated $[A T M]$ ) and $<5 \%$ (e.g. myeloid differentiation primary response 88 [MYD88], baculoviral IAP repeat-containing protein 3 [BIRC3]) frequencies. These new mutations could partly explain the CLL heterogeneity and help in identifying clinically relevant groups of patients [8-11]. In the case of NOTCH1, mutations were detected more often among patients with progressive or high-risk CLL, while the role of MYD88 has not yet been fully elucidated [12, 13]. Rossi et al. [14] believed that integration of these new mutational disorders with cytogenetic model results in more precise prediction of survival compared to the Döhner model alone. The 2016 revision of the World Health Organization classification suggested that these novel mutations have a potential clinical relevance and could be integrated into an updated cytogenetic risk profile [15]. However, a prognostic model created in the same year by the International Chronic Lymphocytic Leukemia-International Prognostic Index (CLL-IPI) Working Group included only IGVH and TP53 molecular mutations, recognizing the other mutations as showing no independent prognostic value. Therefore, there is a need to verify clinical significance of other novel prognostic factors [16].

The NOTCH1 (neurogenic locus notch homolog protein 1) gene, encoded on chromosome 9q34.3, takes a fundamental biological role in hematopoiesis [8]. NOTCH1 receptors have been shown to have an essential role in the pathogenesis of some hematologic and solid malignancies $[17,18]$. The extracellular domain of NOTCH1 (N-EC) consists of 36 epidermal growth factorlike repeats (EGFR), 3 cysteine-rich lin12/Notch repeats (LNR) and the (HD) heterodimerization domain. The intracellular part (N-IC) includes the RAM (R) domain, ankyrine repeats, transactivation domain (TAD) and the threonine-rich sequence (PEST) that marks N-IC for degradation by FBXW7 [19]. NOTCH1 mutation in CLL patients most frequently concerns $\mathrm{C}$-terminal PEST domain, which takes part in the proteasomal degradation of the activated form of NOTCH1 [20]. In fact, mutated, short PEST domain results in stabilization of the active form of $\mathrm{NOTCH1}$, the molecule impaired degradation, and thus upregulated NOTCH1 signaling [21, 22]. NOTCH1 mutations affect 5-10\% newly diagnosed CLL and 15-20\% in progressive CLL requiring first treatment and in relapsed cases [23, 24]. This mutation is associated with more aggressive clinical presentation of CLL such as chemorefractoriness and disease progression towards transformation into RS. NOTCH1 mutations in RS occur in approximately $30 \%$ of cases and are the second, after TP53, most frequent genetic lesions $[8,23]$. Studies on more numerous and specific subgroups of patients have revealed a particularly high frequency of NOTCH1 mutations in CLL cases that harbor trisomy $12(+12)$ as the sole cytogenetic abnormality (30\%) [25]. Moreover, patients with NOTCH1 mutations present a significantly shorter overall survival refining the intermediate prognosis of CLL patients with +12 . Importantly, the high frequency of $\mathrm{NOTCH} 1$ mutations in trisomy 12 CLL patients is associated with a characteristic gene-expression profile characterized by an overexpression of cell cycle-related 
genes inserted on chromosome 12 [25]. NOTCH1 mutations may represent potential new biomarker for the selection of poor-risk CLL patients. Patients with NOTCH1 mutations are characterized by a significantly shorter OS (21-45\% at 10 years) and present a more rapidly progressive disease compared to NOTCH1 wild-type cases (56-66\% at 10 years) $[8,23]$. According to Mansouri et al., NOTCH1 mutations similarly as TP53 mutations seem to be strong, independent prognostic markers of poor prognosis [26].

Myeloid differentiation primary response 88 (MYD88) is a protein that plays a key role in the innate and adaptive immune response and is encoded by the MYD88 gene locating on the short arm of chromosome 3 (p) at position 22 (3p22) [27]. MYD88 acts as a signaling adaptor protein that activates the NF-kB pathway after stimulation of toll-like receptors (TLRs) and receptors for IL-1 and IL-18 on independent and dependent signaling pathways [28]. Moreover, MYD88 co-ordinates the gathering of a multi-subunit signaling complex consisting of various members of the IRAK family of serine-threonine kinases [29]. The most common mutation is a single-nucleotide change (c.794T.C) that leads to switching of leucine to proline at codon 265 (p.L265P) [30]. That dominant mutation triggers constitutive NF-kB stimulation, thus conferring a proliferation and survival advantage to the mutant cells. Ngo et al. described mutations in MYD88 in 39\% of patients of (ABCDLBCL) activated $B$ cell type diffuse large $B$ cell lymphoma, with a single L265P substitution accounting for $75 \%$ of the mutations [31]. The L265P mutation occurs in $2-10 \%$ of cases of CLL $[8,30]$ and almost $100 \%$ of cases of Waldenström's macroglobulinemia [32]. MYD88 mutations in CLL patients are strikingly enriched among (M-CLL) patients expressing mutated $I G H V$ genes [33]. Baliakas et al. [34] in a collaborative multicenter series of 1039 well-annotated CLL cases identified MYD88 mutations in 24/1080 (2.2\%) CLL patients and $92 \%$ cases implemented the hotspot p.L265P substitution. Xia et al. [33] in a study on Chinese population with CLL, found MYD88 mutations (exons 3-5) in 23 of 295 (8\%) analyzed cases. These mutations were more common in patients with mutated $I G V H$ (2/115 vs. $21 / 172$; $\mathrm{p}=0.001)$. In another study, Jeromin et al. [35] investigated a large cohort of 1160 untreated CLL patients for novel prognostic markers considering MYD88. This mutation was detected in 15 of 969 cases $(1.5 \%)$ and it was associated with mutated $I G V H$ status.

\section{Material and methods}

\section{Characteristics of patients}

The research material was peripheral blood and bone marrow of CLL patients. The study included 200 newly diagnosed and previously untreated patients with CLL. The NOTCH1 $(\mathrm{n}=200)$ cohort included 88 women and 112 men at a median age of 66 years (range, $42-90$ years). The MYD88 $(\mathrm{n}=60)$ cohort included 38 women and 22 men at a median age of 67 years (range, $50-84$ years). Distribution of disease stages according to the Rai classification in NOTCH1 group was as follows: stage 0, 63 patients; stage I, 33 patients; stage II, 48 patients; stage III, 12 patients; and stage IV, 20 patients. Distribution of disease stages according to the Rai classification in MYD88 group was as follows: stage 0,18 patients; stage I, 10 patients; stage II, 14 patients; stage III, 4 patients; and stage IV, 8 patients.

Additionally, expression of MYD88 in CLL was assessed in peripheral blood $(\mathrm{PB})(\mathrm{n}=60)$ and bone marrow $(\mathrm{BM})(\mathrm{n}=92)$ of CLL patients. The detailed clinical characteristics of patients are presented in Table 1.

Ethical approval was granted by local review committees and informed consent was collected according to the principles laid by the Declaration of Helsinki. The approval number of the Ethical Commission is KE-0254/231/2015.

\section{Peripheral blood mononuclear cells and DNA isolation}

Peripheral blood mononuclear cells (PBMCs) from CLL patients were isolated by Ficoll density gradient centrifugation (Biochrom, Berlin, Germany). For DNA preparation form PBMCs, QIAamp DNA Blood Mini Kit (Qiagen, Hilden, Germany) was used according to the manufacturer's instructions. DNA was quantified by BioSpec-nano (Shimadzu, Kyoto, Japan).

\section{Amplification refractory mutation system for NOTCH1 and MYD88 mutations}

MYD88 L265P $(\mathrm{n}=60)$ and NOTCH1 c.7544_7545delCT $(\mathrm{n}=200)$ in the PEST domain (exon 34) mutations were investigated by (ARMS-PCR) amplification refractory mutation system polymerase chain reaction. Reactions for NOTCH1 were performed in a $20 \mu \mathrm{l}$ reaction volume containing the following: primer for the wildtype gene sequence, primer for the mutated gene sequence, reverse primer and multiplex. Reactions for MYD88 were performed in 2 samples, a $20 \mu \mathrm{l}$ 
Table 1. Clinical characteristic of CLL patients

\begin{tabular}{|c|c|c|}
\hline Characteristic & $\begin{array}{l}\text { NOTCH1 } \\
\text { mutation }\end{array}$ & $\begin{array}{c}\text { MYD88 } \\
\text { mutation }\end{array}$ \\
\hline Number of patients & 200 & 60 \\
\hline \multicolumn{3}{|l|}{ Sex } \\
\hline Females & 88 & 38 \\
\hline Males & 112 & 22 \\
\hline Age median (range) & $66(42-90)$ & $67(50-84)$ \\
\hline \multicolumn{3}{|l|}{ Rai stage } \\
\hline 0 & 63 & 18 \\
\hline 1 & 33 & 10 \\
\hline 2 & 48 & 14 \\
\hline 3 & 12 & 4 \\
\hline 4 & 20 & 8 \\
\hline Not available & 24 & 6 \\
\hline \multicolumn{3}{|l|}{ ZAP-70 (cut-off 20\%) } \\
\hline Positive & 53 & 12 \\
\hline Negative & 102 & 40 \\
\hline Not available & 45 & 8 \\
\hline \multicolumn{3}{|l|}{ CD38 (cutt-off 30\%) } \\
\hline Positive & 53 & 11 \\
\hline Negative & 111 & 42 \\
\hline Not available & 36 & 7 \\
\hline \multicolumn{3}{|l|}{ IGVH mutation status } \\
\hline Mutated & 76 & 20 \\
\hline Unmutated & 67 & 31 \\
\hline Not available & 57 & 9 \\
\hline
\end{tabular}

reaction volume each; the first sample contained multiplex, forward primer, and reverse primer for the wild-type gene; the second sample contained multiplex, forward primer, and reverse primer for the mutated gene. ARMS-PCR products were separated by gel electrophoresis.

mRNA preparation, reverse transcription and quantitative reverse transcriptase-polymerase chain reaction for MYD88 expression

For total RNA isolation from PBMCs QIAamp RNA Blood Mini Kit (Qiagen, Venlo, Netherlands) was used according to the manufacturer's instructions. From each sample, $1 \mu \mathrm{g}$ of total RNA was reverse transcribed to $20 \mu \mathrm{l}$ of cDNA using QuantiTect Reverse Transcription Kit (Qiagen). For quantitative RT-PCR reactions, $1 \mu \mathrm{l}$ of cDNA of each sample was used.

Expression of MYD88 in CLL was assessed in PB $(n=60)$ and BM $(n=92)$ of CLL patients and 25 healthy volunteers (HVs) using (qRT-PCR) quan- titative reverse transcriptase-polymerase chain reaction. qRT-PCR was performed using TaqMan Gene Expression Assays methodology according to the manufacturer protocol (Applied Biosystems, Foster City). As a constitutively expressed housekeeping gene, $(G A P D H)$ glyceraldehyde-3-phosphate dehydrogenase was used. Thermocycling program was set for 40 cycles of $15 \mathrm{sec}$ at $95^{\circ} \mathrm{C}$, $1 \mathrm{~min}$ at $60^{\circ} \mathrm{C}$ on the ABI Prism 7300 Sequence Detector (Applied Biosystems). Expression levels were calculated as an inverse ratio of the difference in cycle threshold $(\Delta \mathrm{Ct})$, where $\Delta \mathrm{Ct}$ is the $\mathrm{Ct}$ value of the target receptors minus $\mathrm{Ct}$ value of $G A P D H$.

Flow cytometry analysis for ZAP-70 and CD38

The expression of ZAP-70 and CD-38 was assessed by flow cytometry. Based on a ZAP-70 cut-off value of $20 \%$, patients with CLL were categorized into a ZAP-70-positive $(\geq 20 \%)$ or a ZAP-70-negative subgroup $(<20 \%)$. For CD-38, the cut-off value was defined as $30 \%$ and patients were categorized into a CD-38-positive $(\geq 30 \%)$ or a CD-38-negative subgroup $(<30 \%)$.

\section{Statistical analysis}

Statistical analyses were performed using GraphPad Prism 5 (La Jolla, California, United States). The Mann-Whitney and Kruskal-Wallis tests were used to evaluate the differences between the subgroups. The correlations of variables were computed with the Spearman rank correlation coefficient.

\section{Results}

\section{NOTCH1 mutations}

NOTCH1 c.7544_7545delCT mutation occurred in 18 of 200 patients (9\%) with CLL. Patients harboring the NOTCH1 mutation prevalently belonged to aggressive cases, i.e. were significantly more often characterized by the unmutated $I G V H$ gene status ( $\mathrm{n}=17$ of $18,94 \%$ ) than patients with the wild-type NOTCH1 gene $(\mathrm{n}=50$ of $112,44 \%) ;(\mathrm{p}=0.0001)$. The mutation was significantly more often associated with high level of CD38 expression. It accounted for 10 of 18 CD38-positive cases (55\%) among individuals with the NOTCH1 mutation in comparison with 43 of 141 cases $(30 \%)$ with the wild-type gene $(\mathrm{p}=0.02)$. The expression of ZAP-70 was also significantly higher in individuals with mutated NOTCH1 gene ( $\mathrm{n}=12$ of $18,66 \%)$ than in those with wild-type $N O T C H 1$ gene $(\mathrm{n}=41$ of 136 , $30 \%)(\mathrm{p}=0.0008)$. Representative results of the NOTCH1 ARMS assay are shown in Figure 1. The 


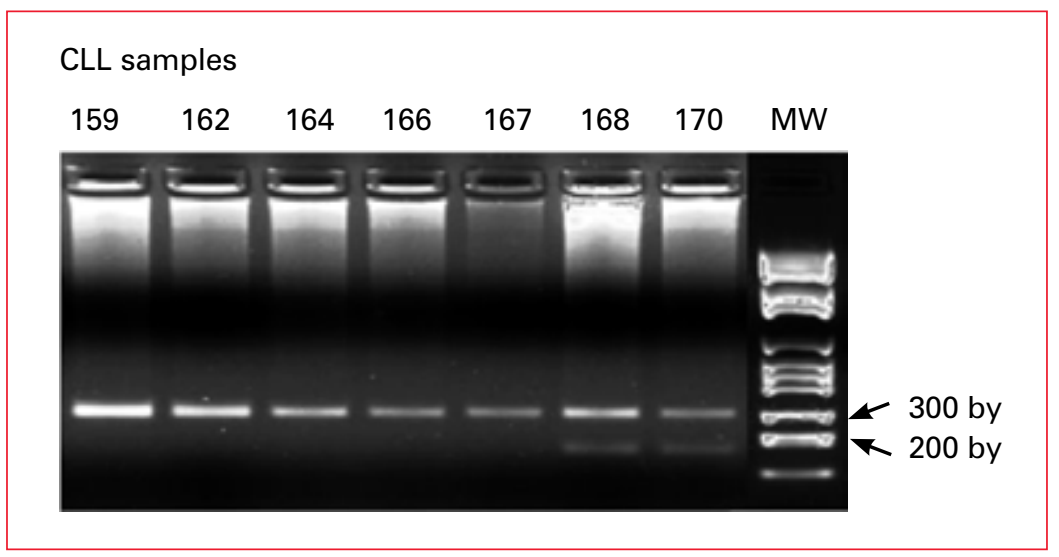

Figure 1. ARMS to detect NOTCH1 c.7544_7545deICT mutation. Representative results of the ARMS assay showing 2 CLL samples that scored positive for the c.7544 7545delCT mutation (codes 168, 170) and 5 CLL samples that scored negative for the c.7544 7545delCT mutation (codes 159, 162, 164, 166, 167). Negative samples show a normal band of $284 \mathrm{bp}$. Positive samples show an additional mutant band of $183 \mathrm{bp}$. Molecular Weight (MW) is the 100-bp DNA ladder

complete analysis of the association between the NOTCH1 mutation and prognostic markers in CLL is presented in Figure 2.

\section{MYD88 mutations}

The MYD88 L265P (exon 5) mutation occurred in 2 of 60 patients (3.3\%) with CLL. MYD88 mutations were strikingly enriched among patients expressing mutated $I G V H$ genes. The median expression of $M Y D 88$ in $\mathrm{PB}(1 / \Delta \mathrm{Ct})$ was 0.178 , in the HVs we observed lower expression of $M Y D 88$ $(1 / \Delta \mathrm{Ct}$ of 0.128$)$. The median expression of $M Y D 88$ in $\mathrm{PB}(1 / \Delta \mathrm{Ct})$ was 0.178 , in the $\mathrm{BM}$ we observed lower expression of $M Y D 88(1 / \Delta \mathrm{Ct}$ of 0.160$)$. These differences reached statistically significance (respectively $\mathrm{p}<0.0001$ and $\mathrm{p}=0.0015$ ). The median expression of MYD88 $(1 / \Delta \mathrm{Ct})$ in $\mathrm{BM}$ was significantly higher in CLL patients with stage Rai I $(0.172)$ than in patients with stage II $(0.152)$ and III (0.155) (respectively $\mathrm{p}=0.03$ and $\mathrm{p}=0.04$ ). There was no correlation between $M Y D 88$ expression in $\mathrm{PB}$ and $\mathrm{BM}$ and expression of ZAP-70, CD38 and IGVH mutational status. Representative results of the MYD88 ARMS assay are shown in Figure 3. Characterization of MYD88 mRNA expression in CLL patients is presented in Figure 4A, $4 \mathrm{~B}$ and $4 \mathrm{C}$.

\section{Discussion}

Thanks to the development of modern methods of molecular biology, the prognostic value of previously unknown factors has been identified and the usefulness of subsequent ones is verified in numerous scientific studies. Because CLL is still an incurable disease, there is a need for

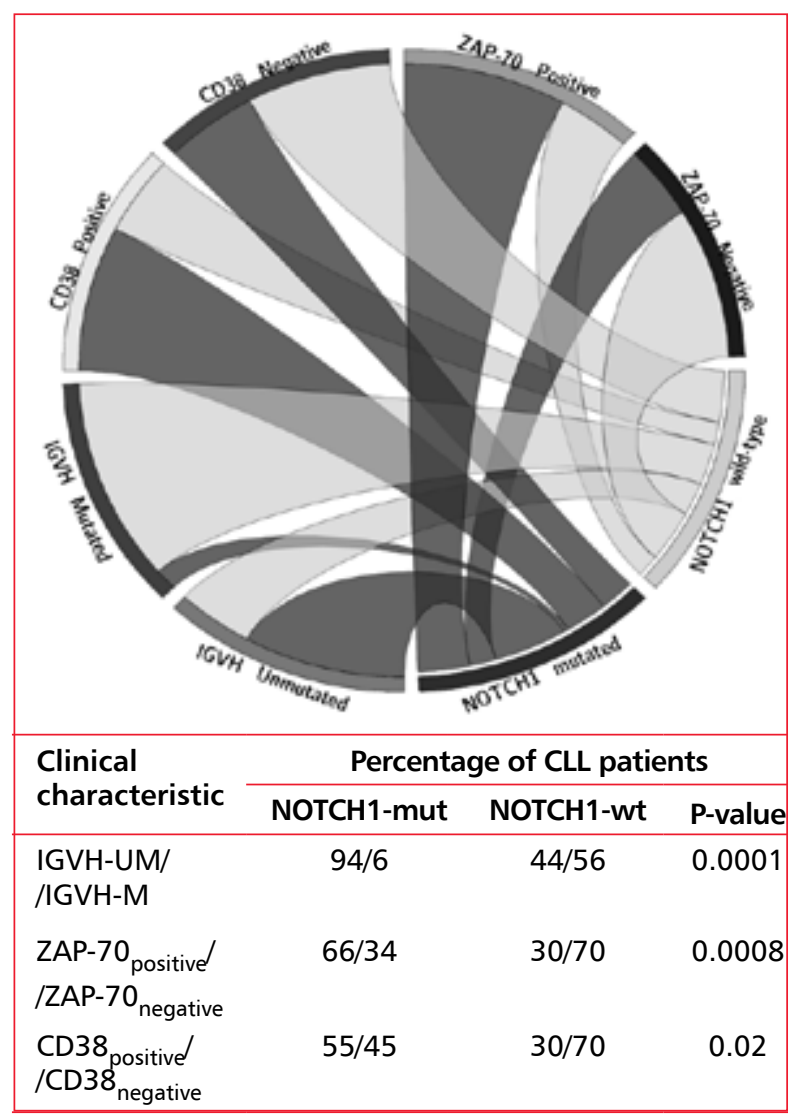

Figure 2. Association between NOTCH1 gene mutation status and clinical characteristic of CLL patients (created using Circos) [36]

research in which the prognostic value of the new factors will be assessed. Since 2000, CLL patients' stratification is workable thanks to the cytogenetic model demonstrated by Döhner et al. 


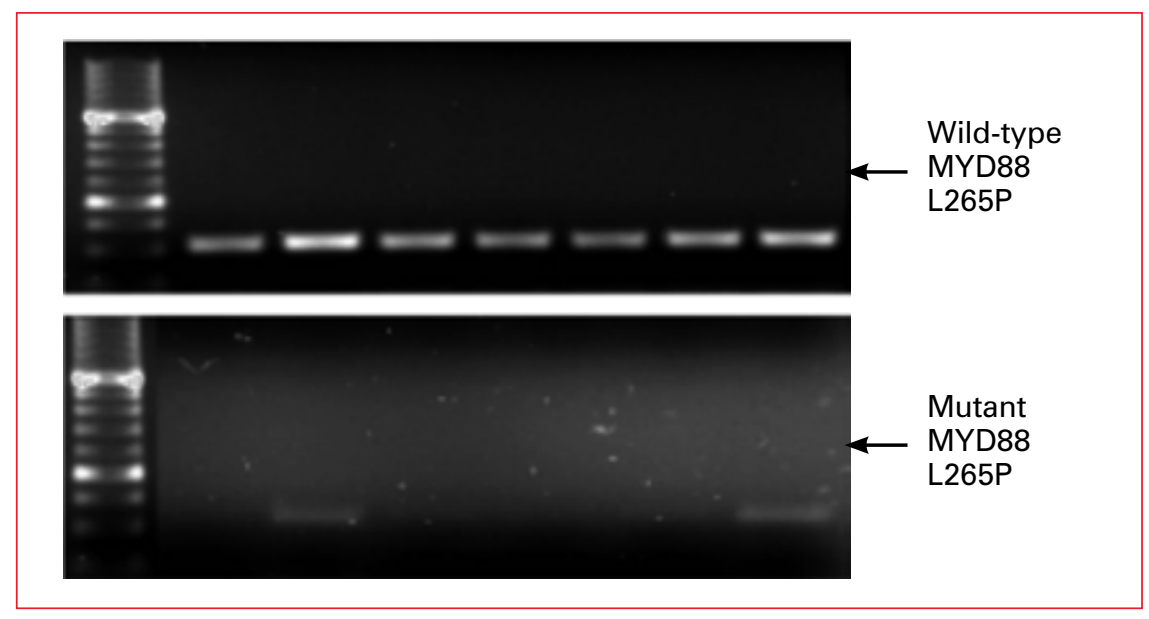

Figure 3. AS-PCR assay for detection of MYD88 L265P. Representative results of the AS-PCR assay showing 2 CLL samples that scored positive for the L265P mutation and 5 CLL samples with MYD88 wild-type sequence

[7]. It concentrates on chromosomal abnormalities, which are detectable in more than $80 \%$ of patients with CLL. Nevertheless, it occurs that molecular lesions provide additional information that also may have prognostic impact. Revision of the classification of lymphoid tumors of the World Health Organization in 2016 showed the potential clinical significance of molecular changes, such as TP53, NOTCH1, SF3B1, ATM and BIRC3 [15]. Some of these changes have been proposed to be included in the updated cytogenetic risk profile, which also contains well-known recurrent chromosomal abnormalities typically identified with fluorescence in situ hybridization studies [34]. In 2016, the International CLL-IPI Working Group created a prognostic model combining biochemical, genetic, and clinical parameters. It includes five independent factors: age, $\beta 2$-microglobulin concentration, clinical stage, IGVH mutation status, and TP53 status. Other than TP53 disorders, recurrent genetic abnormalities, such as NOTCH1 and SF3B1, did not show independent prognostic information [16].

The prognostic impact of the molecular factors such as NOTCH1 and MYD88 has been confirmed by several research groups, but still has not been used in clinical practice $[11-14,34]$. These mutations are thought to be part of the mechanisms explaining the heterogeneity of the disease and may point to new potential therapeutic targets in CLL [11].

To our knowledge, we were the first to assess the occurrence of the analyzed mutations in the cohort of Polish patients. In our study the frequency of the NOTCH1 mutation in newly diagnosed patients with CLL reached $9.0 \%$ and was in agreement with the findings of other investigators [11, 24]. Really, it has been shown that the frequency of mutations can increase as the disease progresses, even up to $20 \%$ during relapse $[9,30]$. The NOTCH1 mutation is associated with especially poor prognosis. The comparison with the presently available prognostic factors revealed the accumulation of negative markers in NOTCH1-mutated individuals. A significant correlation was found with the unmutated $I G V H$ status, confirming the results of previous studies [11, 30, 37-39]. Additionally, the NOTCH1 mutation was significantly more frequently associated with positive expression of CD38 and ZAP-70, which is associated with poor prognosis. Studies revealed that the NOTCH1 mutation was more common among patients in advanced clinical stage, mainly enrolled or treated in clinical trials [34]. NOTCH1 mutation has also a strong positive association with trisomy +12 and negative association with isolated del(13q). What's more, it has been associated with decreased overall survival in Rossi et. al and Oscier et al. studies [11, 24], but was not an independent marker of survival [5].

Our study demonstrated significantly higher PB MYD88 expression than in HVs and relevantly higher PB MYD88 expression in comparison with BM (respectively $\mathrm{p}<0.0001$ and $\mathrm{p}=0.0015$ ). There was no correlation between MYD88 expression in $\mathrm{PB}$ and $\mathrm{BM}$ and expression of ZAP-70, CD38 and $I G V H$ mutational status. Apart from MYD88 expression in current study we also investigated MYD88 mutations. These mutations occurred in 3.3\% CLL patients and were strikingly enriched among patients expressing mutated $I G V H$ genes. Our data argue with previous reports by Xia et al. that demonstrated more common occurrence $M Y D 88$ mutations in patients with mutated $I G V H$ 


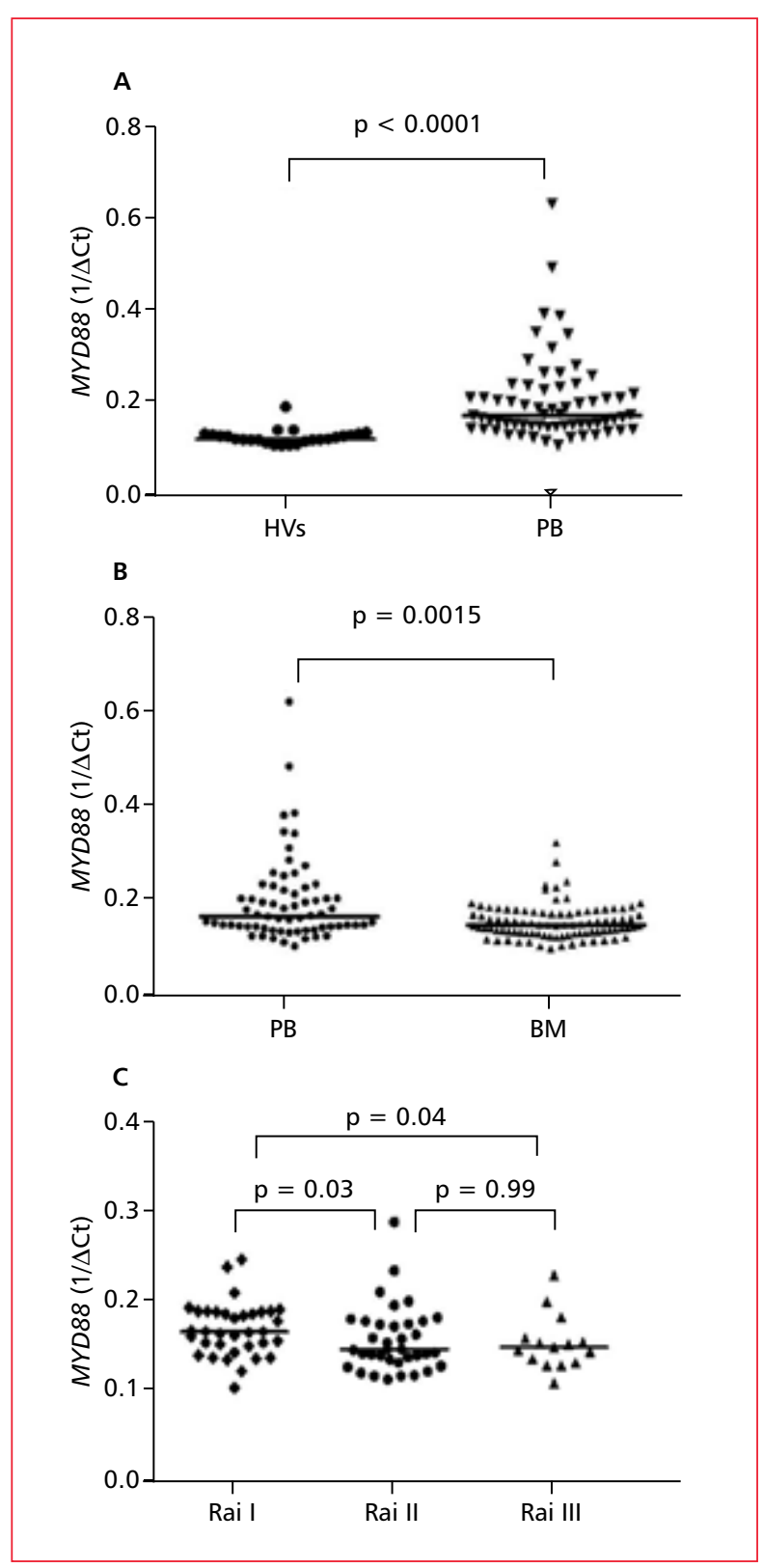

Figure 4. Characterization of MYD88 mRNA expression in chronic lymphocytic leukemia (CLL) patients. A. Expression of MYD88 in peripheral blood of healthy volunteers (HVs) and CLL patients; B. Expression of MYD88 in peripheral blood (PB) and bone marrow (BM) of CLL patients; C. Expression of MYD88 in BM of CLL patients according to the stage of the disease

[33]. The study of Jeromin et al. on the large cohort of CLL patients revealed MYD88 mutations in $1.5 \%$ cases and was also associated with mutated $I G V H$ status [35]. Therefore, the relevance of the MYD88 mutation as a prognostic factor remains unclear and larger collaborative studies are required.

\section{Conclusions}

In conclusion, NOTCH1 mutations are associated with certain, unfavorable biological markers and seem to be independent predictive markers for worse outcome in CLL patients. Definitely, these mutations may help the identification of patients with high-risk CLL, and in combination with established negative prognostic factors of the CLL-IPI, they might provide additional information on disease prognosis. Further collaborative studies in CLL are obligate to study the prognostic and predictive relevance of $M Y D 88$ mutations and expression.

\section{References}

1. Chiorazzi N, Rai KR, Ferrarini M. Chronic lymphocytic leukemia. N Engl J Med. 2005; 352(8): 804-815, doi: 10.1056/ NEJMra041720, indexed in Pubmed: 15728813.

2. Abrisqueta P, Pereira A, Rozman C, et al. Improving survival in patients with chronic lymphocytic leukemia (1980-2008): the Hospital Clinic of Barcelona experience. Blood. 2009; 114(10): 2044-2050, doi: 10.1182/blood-2009-04-214346, indexed in Pubmed: 19553638.

3. Shanafelt TD, Jenkins G, Call TG, et al. Validation of a new prognostic index for patients with chronic lymphocytic leukemia. Cancer. 2009; 115(2): 363-372, doi: 10.1002/cncr.24004, indexed in Pubmed: 19090008.

4. Hallek M, Cheson BD, Catovsky D, et al. International Workshop on Chronic Lymphocytic Leukemia. Guidelines for the diagnosis and treatment of chronic lymphocytic leukemia: a report from the International Workshop on Chronic Lymphocytic Leukemia updating the National Cancer Institute-Working Group 1996 guidelines. Blood. 2008; 111(12): 5446-5456, doi: 10.1182/ blood-2007-06-093906, indexed in Pubmed: 18216293.

5. Dighiero G, Hamblin TJ. Chronic lymphocytic leukaemia. The Lancet. 2008; 371(9617): 1017-1029, doi: 10.1016/s01406736(08)60456-0.

6. Rassenti LZ, Huynh L, Toy TL, et al. ZAP-70 compared with immunoglobulin heavy-chain gene mutation status as a predictor of disease progression in chronic lymphocytic leukemia. N Engl J Med. 2004; 351(9): 893-901, doi: 10.1056/NEJMoa040857, indexed in Pubmed: 15329427.

7. Döhner H, Stilgenbauer S, Benner A, et al. Genomic aberrations and survival in chronic lymphocytic leukemia. N Engl J Med. 2000; 343(26): 1910-1916, doi: 10.1056/NEJM200012283432602, indexed in Pubmed: 11136261.

8. Fabbri G, Rasi S, Rossi D, et al. Analysis of the chronic lymphocytic leukemia coding genome: role of NOTCH1 mutational activation. J Exp Med. 2011; 208(7): 1389-1401, doi: 10.1084/ jem.20110921, indexed in Pubmed: 21670202.

9. Quesada V, Conde L, Villamor N, et al. Exome sequencing identifies recurrent mutations of the splicing factor SF3B1 gene in chronic lymphocytic leukemia. Nat Genet. 2011; 44(1): 47-52, doi: 10.1038/ng.1032, indexed in Pubmed: 22158541.

10. Sportoletti P, Baldoni S, Cavalli L, et al. NOTCH1 PEST domain mutation is an adverse prognostic factor in B-CLL. Br J Haematol. 2010; 151(4): 404-406, doi: 10.1111/j.1365-2141.2010.08368.x, indexed in Pubmed: 20813007. 
11. Rossi D, Rasi S, Fabbri G, et al. Mutations of NOTCH1 are an independent predictor of survival in chronic lymphocytic leukemia. Blood. 2011; 119(2): 521-529, doi: 10.1182/blood-2011-09-379966.

12. Cortese D, Sutton LA, Cahill N, et al. On the way towards a,CLL prognostic index': focus on TP53, BIRC3, SF3B1, NOTCH1 and MYD88 in a population-based cohort. Leukemia. 2014; 28(3): 710-713, doi: 10.1038/leu.2013.333, indexed in Pubmed: 24217197.

13. Del Giudice I, Rossi D, Chiaretti S, et al. NOTCH1 mutations in +12 chronic lymphocytic leukemia (CLL) confer an unfavorable prognosis, induce a distinctive transcriptional profiling and refine the intermediate prognosis of +12 CLL. Haematologica. 2012; 97(3): 437-441, doi: 10.3324/haematol.2011.060129, indexed in Pubmed: 22207691.

14. Rossi D, Rasi S, Spina V, et al. Integrated mutational and cytogenetic analysis identifies new prognostic subgroups in chronic lymphocytic leukemia. Blood. 2013; 121(8): 1403-1412, doi: 10.1182/blood-2012-09-458265, indexed in Pubmed: 23243274.

15. Swerdlow SH, Campo E, Pileri SA, et al. The 2016 revision of the World Health Organization classification of lymphoid neoplasms. Blood. 2016; 127(20): 2375-2390, doi: 10.1182/ blood-2016-01-643569, indexed in Pubmed: 26980727.

16. International CLL-IPI working group. An international prognostic index for patients with chronic lymphocytic leukaemia (CLL-IPI): a meta-analysis of individual patient data. Lancet Oncol. 2016; 17(6): 779-790, doi: 10.1016/S1470-2045(16)30029-8, indexed in Pubmed: 27185642.

17. Rosati E, Sabatini R, Rampino G, et al. Constitutively activated Notch signaling is involved in survival and apoptosis resistance of B-CLL cells. Blood. 2009; 113(4): 856-865, doi: 10.1182/ blood-2008-02-139725, indexed in Pubmed: 18796623.

18. Hajdu M, Sebestyén A, Barna G, et al. Activity of the notch-signalling pathway in circulating human chronic lymphocytic leukaemia cells. Scand J Immunol. 2007; 65(3): 271-275, doi: 10.1111/j.1365-3083.2006.01897.x, indexed in Pubmed: 17309782.

19. Gianfelici V. Activation of the NOTCH1 pathway in chronic lymphocytic leukemia. Haematologica. 2012; 97(3): 328-330, doi: 10.3324/haematol.2012.061721, indexed in Pubmed: 22383743.

20. Di Ianni M, Baldoni S, Rosati E, et al. A new genetic lesion in B-CLL: a NOTCH1 PEST domain mutation. Br J Haematol. 2009; 146(6): 689-691, doi: 10.1111/j.1365-2141.2009.07816.x, indexed in Pubmed: 19604236.

21. Paganin M, Ferrando A. Molecular pathogenesis and targeted therapies for NOTCH1-induced T-cell acute lymphoblastic leukemia. Blood Rev. 2011; 25(2): 83-90, doi: 10.1016/j.blre.2010.09.004, indexed in Pubmed: 20965628.

22. Pear WS, Aster JC. T cell acute lymphoblastic leukemia/lymphoma: a human cancer commonly associated with aberrant NOTCH1 signaling. Curr Opin Hematol. 2004; 11(6): 426-433, indexed in Pubmed: 15548998.

23. Rossi D, Rasi S, Fabbri G, et al. Mutations of NOTCH1 are an independent predictor of survival in chronic lymphocytic leukemia. Blood. 2011; 119(2): 521-529, doi: 10.1182/blood-2011-09-379966.

24. Oscier DG, Rose-Zerilli M, Winkelmann N, et al. The clinical significance of NOTCH1 and SF3B1 mutations in the UK LRF CLL4 trial. Blood. 2012; 121(3): 468-475, doi: 10.1182/ blood-2012-05-429282.

25. Del Giudice I, Rossi D, Chiaretti S, et al. NOTCH1 mutations in +12 chronic lymphocytic leukemia (CLL) confer an unfavorable prognosis, induce a distinctive transcriptional profiling and refine the intermediate prognosis of +12 CLL. Haematologica. 2012; 97(3): 437-441, doi: 10.3324/haematol.2011.060129, indexed in Pubmed: 22207691.

26. Mansouri L, Cahill N, Gunnarsson R, et al. NOTCH1 and SF3B1 mutations can be added to the hierarchical prognostic classification in chronic lymphocytic leukemia. Leukemia. 2013; 27(2): 512-514, doi: 10.1038/leu.2012.307, indexed in Pubmed: 23138133.

27. Amaya-Chanaga CI, Rassenti LZ. Biomarkers in chronic lymphocytic leukemia: Clinical applications and prognostic markers. Best Pract Res Clin Haematol. 2016; 29(1): 79-89, doi: 10.1016/j. beha.2016.08.005, indexed in Pubmed: 27742074.

28. Iwasaki A, Medzhitov R, Iwasaki A, et al. Regulation of adaptive immunity by the innate immune system. Science. 2010; 327(5963): 291-295, doi: 10.1126/science.1183021, indexed in Pubmed: 20075244.

29. Vandenbon A, Teraguchi S, Akira S, et al. Systems biology approaches to toll-like receptor signaling. Wiley Interdiscip Rev Syst Biol Med. 2012; 4(5): 497-507, doi: 10.1002/wsbm.1178, indexed in Pubmed: 22714995.

30. Wang JQ, Jeelall YS, Ferguson LL, et al. Toll-Like Receptors and Cancer: MYD88 Mutation and Inflammation. Front Immunol. 2014; 5: 367, doi: 10.3389/fimmu.2014.00367, indexed in Pubmed: 25132836.

31. Ngo VuN, Young RM, Schmitz R, et al. Oncogenically active MYD88 mutations in human lymphoma. Nature. 2011; 470(7332): 115-119, doi: 10.1038/nature09671, indexed in Pubmed: 21179087.

32. Treon SP, Xu L, Yang G, et al. MYD88 L265P somatic mutation in Waldenström's macroglobulinemia. N Engl J Med. 2012; 367(9): 826833, doi: 10.1056/NEJMoa1200710, indexed in Pubmed: 22931316.

33. Xia Yi, Fan L, Wang Li, et al. Frequencies of SF3B1, NOTCH1, MYD88, BIRC3 and IGHV mutations and TP53 disruptions in Chinese with chronic lymphocytic leukemia: disparities with Europeans. Oncotarget. 2015; 6(7): 5426-5434, doi: 10.18632/ oncotarget.3101, indexed in Pubmed: 25605254.

34. Baliakas P, Hadzidimitriou A, Sutton LA, et al. European Research Initiative on CLL (ERIC). Recurrent mutations refine prognosis in chronic lymphocytic leukemia. Leukemia. 2015; 29(2): 329336, doi: 10.1038/leu.2014.196, indexed in Pubmed: 24943832.

35. Jeromin S, Weissmann S, Haferlach C, et al. SF3B1 mutations correlated to cytogenetics and mutations in NOTCH1, FBXW7, MYD88, XPO1 and TP53 in 1160 untreated CLL patients. Leukemia. 2014; 28(1): 108-117, doi: 10.1038/leu.2013.263, indexed in Pubmed: 24113472.

36. Krzywinski M, Schein J, Birol I, et al. Circos: an information aesthetic for comparative genomics. Genome Res. 2009; 19(9): 16391645, doi: 10.1101/gr.092759.109, indexed in Pubmed: 19541911.

37. Chiaretti S, Marinelli M, Giudice ID, et al. NOTCH1, SF3B1, BIRC3andTP53mutations in patients with chronic lymphocytic leukemia undergoing first-line treatment: correlation with biological parameters and response to treatment. Leukemia \& Lymphoma. 2014; 55(12): 2785-2792, doi: 10.3109/10428194.2014.898760.

38. Ramsay AJ, Quesada V, Foronda M, et al. POT1 mutations cause telomere dysfunction in chronic lymphocytic leukemia. Nat Genet. 2013; 45(5): 526-530, doi: 10.1038/ng.2584, indexed in Pubmed: 23502782.

39. Puente XS, Pinyol M, Quesada V, et al. Whole-genome sequencing identifies recurrent mutations in chronic lymphocytic leukaemia. Nature. 2011; 475(7354): 101-105, doi: 10.1038/nature10113, indexed in Pubmed: 21642962. 